\title{
On Optimal Recovery from Terminal Understeer
}

\author{
Matthijs Klomp*, eAAM Drivline Systems \\ Mathias Lidberg, Chalmers University of Technology \\ Tim J. Gordon, University of Michigan
}

August 28, 2013

\begin{abstract}
This paper addresses the problem of terminal understeer and its mitigation via integrated brake control. The scenario considered is when a vehicle enters a curve at a speed that is too high for the tyre/road friction limits and an optimal combination of braking and cornering forces is required to slow the vehicle down and negotiate the curve. Here, the driver commands a step steering input, from which a circular arc reference path is inferred. An optimal control problem is formulated with an objective to minimize the maximum off-tracking from the reference path and two optimal control solutions are obtained. The first is an explicit analytic solution for a friction-limited particle; the second is a numerically derived open-loop brake control sequence for a nonlinear vehicle model. The particle solution is found to be a classical parabolic trajectory associated with a constant global mass-center acceleration vector. The independent numerical optimization for the vehicle model is found to closely approximate the
\end{abstract}

*Corresponing author's e-mail: matthijs.klomp@aam.com 
kinematics of the parabolic path reference (PPR) strategy obtained for the particle. Using the PPR strategy, a closed-loop controller is formulated and verified against the solution from numerical optimization. Results are further compared to understeer mitigation by yaw control ( $\mathrm{YC}$ ) and the PPR controller is found to give significant improvement over $\mathrm{YC}$ for this scenario.

\section{Introduction}

Vehicle road holding and yaw stability are both essential for safe driving. We use the term "road holding" in the sense of path control - the ability to adjust the curvature of a vehicle's trajectory by regulating speed and path-lateral acceleration. The available control actuators are assumed to be front wheel steering commanded by the driver, plus individual wheel braking commanded by an on-board control system. In this paper we consider the terminal understeer problem, where the available path-lateral acceleration is not sufficient for the vehicle to follow a desired path. This is recognized in accident statistics [1, 2] as a situation where curve entry speed is too high for the required path curvature, given the prevailing tyre/road friction; as a result the vehicle follows a wider path than desired, incurring multiple risks associated with unintended lane or road departure. To minimize such risk, it is proposed to apply brake forces for "optimal recovery" from terminal understeer, which we formulate as the minimization of off-tracking. More specifically, intended path curvature is inferred from the driver's steering input and the control system is to minimize the maximum deviation from this path. We note that while overall yaw stability should be maintained, the control target is defined in terms of path deviation. 
By contrast to understeer, terminal oversteer mitigation is associated with recovery from yaw instability, and has received a great deal of attention in the literature [3, 4, 5, 6, 7. Yaw instability is suppressed by the application of braking forces to generate yaw moments acting in the opposite sense to the direction of turn, simultaneously reducing excessive yaw rate and body sideslip. Some authors make the assumption that understeer mitigation is the direct opposite of oversteer mitigation, and hence the same type of yaw control (YC) by differential braking is the appropriate intervention; now the yaw moment should be applied in the same sense as the direction of turn, in order to increase yaw rate and potentially reduce understeer [6, 7. In this approach, while it is true that the applied yaw moment will increase the vehicle heading angle in the turn direction, there is no guarantee that it will also increase path curvature, especially when operating at the limits of friction. Furthermore, there is the potential to induce yaw instability, requiring a subsequent oversteer intervention.

Alternative approaches to understeer mitigation have been attempted by other authors. In [8] the problem of understeer is addressed by a combination of speed reduction and yaw moment control. The proposed control reduces vehicle speed during the turn-in phase of the maneuver, braking being proportional to lateral jerk. While it is reported that this control reduces the effects of understeer, no formal control objective is defined. In an earlier study 9 the focus was again on modulating the direction of the mass center acceleration vector, here with a more explicit focus on using combined cornering and braking forces to increase the path curvature.

Previous work by the authors in [10] includes initial numerical optimal control results and in [11] promising experimental results with a preliminary closed- 
loop controller for improved understeer mitigation. We seek to build on this research and develop a new and fundamental approach to the problem of understeer mitigation. In the following we formulate the problem of combined speed and directional control to minimize off-tracking when curve entry speed is too high, or equivalently the tyre/road friction is too low. While interaction with the driver is a crucial part of a system of this kind, for clarity we focus on fundamental performance capabilities in the case where the driver's steering input is a step function, and where the inferred target trajectory is a circular arc.

Section 2 defines our two-track nonlinear vehicle model, and Section 3 considers optimal understeer interventions. A simple form of closed-loop control is introduced in Section 4 and comparisons are made with a similar controller that uses turning-in yaw moments [7]. Table 1 summarises the various control schemes used. Conclusions are given in Section 5 .

\begin{tabular}{c|l} 
Acronym & Description \\
\hline YC & Yaw moment control: comparative closed-loop controller \\
OCP & Optimal Control using the Particle model (analytic solution) \\
OCV & Optimal open-loop Control using the Vehicle model (numerical optimization) \\
PPR & Parabolic Path Reference used for closed-loop implementation
\end{tabular}

Table 1: Summary of control strategies used in this paper

\section{Vehicle System Modeling}

In this section a vehicle model is presented for the study of the relevant planar motion of a standard passenger vehicle. The target application is for a real 
vehicle with active brake controls - each wheel is to be capable of individual wheel braking, controlled through solenoid valves, as is typical in a standard antilock braking system. The brake controller will be assumed to have full authority of the braking torque, and have access to wheel speed sensors to assist with low-level modulation of braking torque. Other required sensors are for steering angle, lateral acceleration and yaw rate, all commonly available in commercial stability control systems. Furthermore, an estimation of the road friction is required, but only once the friction limit is reached [11.

The two-track vehicle model is shown in Figure 1. The model was previously used in [12] and it is based on the assumptions found in references [3, 13]. It is a planar model, with suspension motions suppressed, but with load transfer effects considered. The model assumes front steering with equal angles at the left and right wheels. The longitudinal tyre forces $F_{X i j}$ are individually controlled; here $i$ is the index for the front/rear wheels and $j$ for the left/right wheels see Figure 1. Lateral tyre forces, $F_{Y i j}$, are then determined via a nonlinear combined-slip tyre model - see Appendix A

The equations of motion are given below, derived from the standard NewtonEuler theory using motion variables in the vehicle-fixed $X-Y$ reference frame 


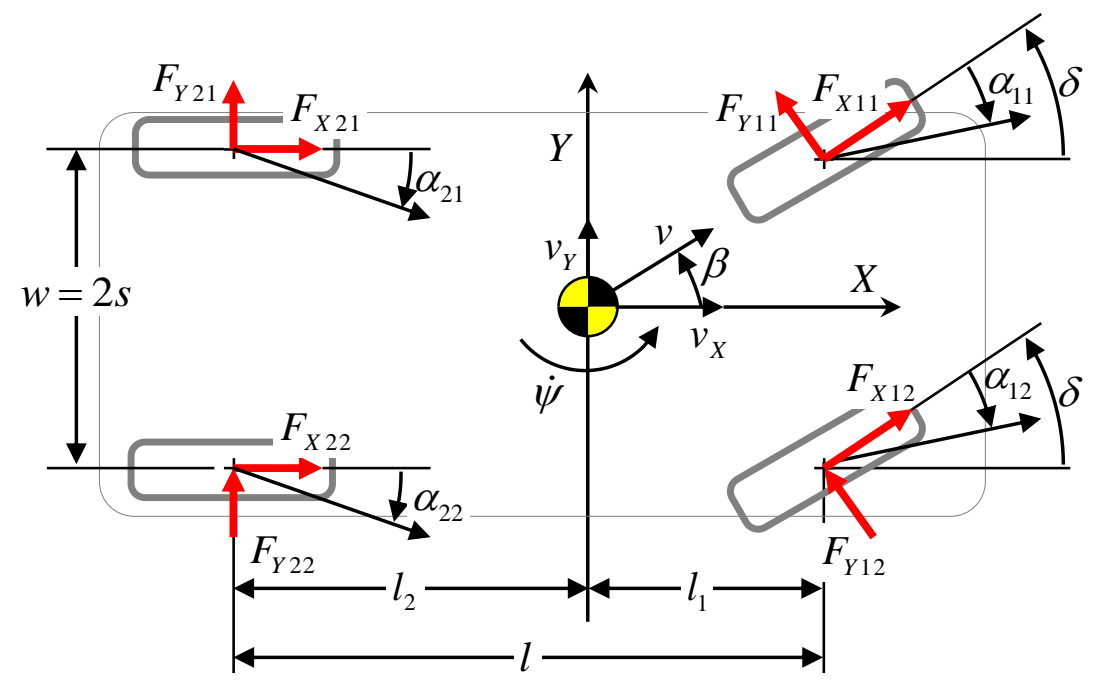

Figure 1: Two-track vehicle model. The arrows show the positive direction of each angle or force.

(see Figure 1) and the inertial $X_{E}-Y_{E}$ reference frame (see Figure 2):

$$
\begin{aligned}
m\left(\dot{v}_{X}-v_{Y} \dot{\psi}\right) & =\sum_{i, j} \bar{F}_{X i j} \\
m\left(\dot{v}_{Y}+v_{X} \dot{\psi}\right) & =\sum_{i, j} \bar{F}_{Y i j} \\
m k^{2} \ddot{\psi} & =\sum_{i, j}\left((-1)^{j} s \bar{F}_{X i j}-(-1)^{i} l_{i} \bar{F}_{Y i j}\right) \\
\dot{X}_{E} & =v_{X} \cos \psi-v_{Y} \sin \psi \\
\dot{Y}_{E} & =v_{X} \sin \psi+v_{Y} \cos \psi
\end{aligned}
$$

where $\bar{F}_{X i j}$ and $\bar{F}_{Y i j}$ are the respective longitudinal and lateral wheel forces resolved in the local vehicle reference frame, $m$ the total vehicle mass and $k$ is the radius of gyration. Vehicle data are given in Appendix B. The vertical forces, $F_{Z i j}$, are a result of the static load distribution and the load transfer due to the longitudinal and lateral acceleration [13. This results in the following 
model for the tyre vertical forces:

$$
F_{Z i j}=\zeta_{0 i} m g+(-1)^{i} \zeta_{X} m a_{X}+(-1)^{j} \zeta_{Y i} m a_{Y}
$$

where, $g, a_{X}$ and $a_{Y}$ are the gravitational, vehicle longitudinal and lateral acceleration, respectively; $\zeta_{0 i}=\left(l-l_{i}\right) /(2 l)$ is the static force distribution coefficient, $\zeta_{X}=h /(2 l)$ is the longitudinal load transfer coefficient, $\zeta_{Y i}$ is the lateral load transfer coefficient of each axle and $h$ is the height of mass center above ground. The lateral load transfer coefficient is a lumped parameter taking the roll stiffness distribution, roll center heights, etc. into account and is listed in Appendix $\mathrm{B}$

\section{Understeer Mitigation}

As discussed in the introduction, in the scenario of interest the driver aims to follow a desired path while maintaining sufficient yaw stability. First we define the inference of desired path from the driver's steering input. The optimal recovery from terminal understeer is then defined as an optimal control problem. A closed-form solution is obtained for a simple particle representation of the vehicle, and then a more realistic solution is obtained via numerical optimization of the vehicle model defined above. Comparisons are then made between the particle and vehicle optimization results.

\subsection{Driver Interpreter and Friction Limits}

It is common to interpret the desired vehicle lateral response from the driver's steering wheel angle $\delta_{H}$ using a reference vehicle model [5, 7]. Here the driver interpreter is based on a very simple reference - a neutral steered vehicle without 
time delay; the desired path curvature $\kappa_{\text {ref }}$ is expressed as follows as a function of the steering wheel input:

$$
\kappa_{\text {ref }}=\frac{\delta_{H}}{i_{S} l}
$$

where $i_{S}$ is the ratio between $\delta_{H}$ and the road wheel angle $\delta$ of the front wheels. While it is possible to include other features in the reference model, such as a preassigned understeer gradient or transient behavior, simplicity of the reference model seems most appropriate for this fundamental analysis.

In order to track the desired path, the speed, $v$, must be less than the maximum achievable speed, $v_{\text {lim }}$, for a given combination of path curvature and available combined friction, $\mu$. This speed is obtained for this curvature when all available friction is utilized perpendicular to the velocity vector, such that

$$
v_{\lim }=\sqrt{\mu g\left|\kappa_{\mathrm{ref}}^{-1}\right|}
$$

If braking is applied in a situation where the curve entry speed, $v_{0}$, is equal to $v_{\text {lim }}$, the path curvature is reduced and the vehicle path will drift to the outside of the desired path. This implies a degree of "loss of path control" at the limit of friction: the driver may increase path curvature by reducing speed, but the required deceleration implies reducing path curvature!

Consider now the case $v_{0}>v_{\text {lim }}$, where the actual path necessarily deviates from the desired path due to terminal understeer. While braking reduces instantaneous path curvature, it also gives an opportunity to increase the mean path curvature due to speed reduction, depending on the duration of the brake intervention. Note that we are not discussing braking before the turn, but applying combined braking and corning forces during the turn. The ideal degree of braking is related to the horizon $[0, T]$ over which brakes are applied to re- 


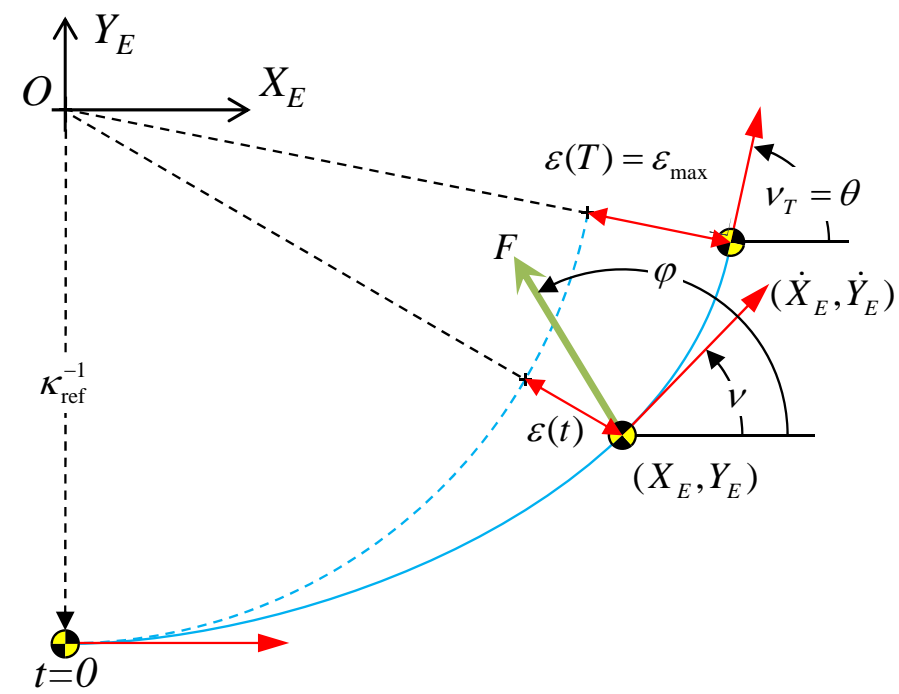

Figure 2: Kinematics of off-tracking due to terminal understeer: vehicle mass center path (solid curve) deviates by $\varepsilon(t)$ from the reference path (dashed curve). Maximum off-tracking $\varepsilon_{\max }$ occurs at time $T$.

duce mean curvature. Intuitively $T$ should increase according to the degree of overspeed, but to avoid fixing $T$ in some ad-hoc manner, control optimization will now be formulated in a way that $T$ is a derived parameter.

\subsection{Recovery from Terminal Understeer as an Optimal Control Problem}

Continuing with the case $v_{0}>v_{\mathrm{lim}}$, the subsequent kinematics of the vehicle path are shown in Figure 2 where the solid curve represents the path of the vehicle mass center and the dashed curve is the circular reference path. The coordinate origin $O$ is chosen at the center of the circle. At time $t=0$ the vehicle is tangent to the reference path, $\dot{X}_{E}=v_{0}$ and $\dot{Y}_{E}=0$. Since $v_{0}>v_{\text {lim }}$, path 
curvature is necessarily less than that of the reference path, and lateral deviation $\varepsilon(t)$ (measured radially from the origin in this case) increases monotonically from zero for $t>0$. Assuming the understeer mitigation is successful, $\varepsilon(t)$ will be bounded and therefore at a later time $T$ it reaches a maximum $\varepsilon_{\max }=\varepsilon(T)$; at this time the radial velocity component is zero, i.e. the vehicle and reference paths are parallel. Subsequent reductions in $\varepsilon(t)$ are then feasible, for example by maintaining constant speed and constant path curvature, so in terms of offtracking the terminal understeer problem is effectively resolved at time $T$.

We now formalize the control objective, which is to minimize the maximum value of $\varepsilon(t)$ in the subsequent motion. Writing the vehicle dynamics model in the following general form,

$$
\dot{\mathbf{x}}=\mathbf{f}(\mathbf{x}, t, \mathbf{u})
$$

the state vector $\mathbf{x}$ includes variables $\left[\begin{array}{llll}X_{E}(t) & Y_{E}(t) & \dot{X}_{E}(t) & \dot{Y}_{E}(t)\end{array}\right]^{\top}$ for the mass center path, and we aim to minimize the cost function

$$
J=X_{E}^{2}(T)+Y_{E}^{2}(T)
$$

where the final time $T$ is free. The state vector is subject to the initial conditions

$$
X_{E}(0)=0, \quad Y_{E}(0)=-\kappa_{\text {ref }}^{-1}, \quad \dot{X}_{E}(0)=v_{0}, \quad \dot{Y}_{E}(0)=0
$$

and the terminal conditions

$$
X_{E}(T) \dot{X}_{E}(T)+Y_{E}(T) \dot{Y}_{E}(T)=0 .
$$

The terminal conditions in Equation (8) ensures that velocity vector at $t=T$ is perpendicular to the position vector of the vehicle relative to $O$ in Figure 2 . This means that the optimal control which minimizes Equation (6), minimizes the maximum off-tracking distance $\varepsilon_{\max }$. 
Friction limits are imposed as constraints on the input variables and optionally there are constraints on the states (e.g. to limit the excursion on sideslip angles):

$$
\begin{aligned}
& \mathbf{u}_{\min } \leq \mathbf{u} \leq \mathbf{u}_{\max } \\
& \mathbf{x}_{\min } \leq \mathbf{x} \leq \mathbf{x}_{\max }
\end{aligned}
$$

In the case of the above vehicle model, the control vector $\mathbf{u}$ comprises the longitudinal tyre forces $F_{X i j}$, while in the next section it is reduced to the resultant force vector acting at the mass-center. Solving the optimal control problem means finding a sequence of admissible control inputs such that the objective function (6) is minimized while satisfying the equations of motion and the initial and final conditions.

\subsection{Optimal Control for a Particle Representation}

As described in Section 3.1 one of the fundamental aspects of terminal understeer is the approximate friction circle exhibited at the vehicle level. Hence we consider the corresponding particle model, where friction limits are imposed but the yaw degree of freedom is suppressed. Although this is a gross simplification of the vehicle dynamics, the particle model offers further insight into understeer mitigation. It also allows the optimal recovery strategy to be determined analytically.

The equations of motion for a particle representation of the vehicle motion are expressed in the inertial reference frame as follows:

$$
\begin{gathered}
\ddot{X}_{E}=(F / m) \cos \varphi \\
\ddot{Y}_{E}=(F / m) \sin \varphi
\end{gathered}
$$


with the set of admissible controls being the magnitude and global direction of the resultant force vector:

$$
\mathcal{U}=\{F \in[0, \mu m g], \varphi \in[0,2 \pi]\}
$$

The optimal control problem is solved in Appendix C where the optimal control input $\left(F^{*}(t), \varphi^{*}(t)\right)$ is shown to be:

$$
\begin{aligned}
F^{*}(t) & =\mu m g \\
\varphi^{*}(t) & =\frac{\pi}{2}+\theta
\end{aligned}
$$

where

$$
\cos \theta=\cos \nu_{T}=\frac{v_{\lim }^{2}}{v_{0}^{2}}
$$

(see also Figure 2). Further, the time of maximum off-tracking under optimal control is found to be

$$
T^{*}=\frac{v_{0} \sin \theta}{\mu g}
$$

The speed of the particle is obtained by integrating Equation (11) with the optimal control input Equation (13) to obtain

$$
v(t)=\sqrt{v_{0}^{2}-2 v_{0} \mu g t \sin \theta+(\mu g t)^{2}}
$$

When combined with Equation (15) this gives

$$
v\left(T^{*}\right)=v_{0} \cos \theta=\frac{v_{\lim }^{2}}{v_{0}}
$$

which is a simple and useful result implying a target terminal speed at time $T^{*}$; this is used below to develop a candidate controller designed to emulate the optimal PPR strategy.

As mentioned above, there exists a control input for $t>T^{*}$ that monotonically decreases the off-tracking distance, namely to maintain constant speed 
$v\left(T^{*}\right)$ and constant curvature, since $v\left(T^{*}\right)<v_{\lim }$ and the terminal path curvature is greater than $\kappa_{\text {ref }}$. Another option is to continue to apply Equation 13 for $t>T^{*}$. It can be verified that this input continues the original parabola symmetrically about $\left(X_{E}(T), Y_{E}(T)\right)$, and returns the path tangentially to the original reference circle. This converging path can be achieved at constant speed, and hence avoid further off-tracking when the reference path is regained. This final part of the path recovery is described only for completeness; as mentioned above, once maximum off-tracking is reached, the intervention is considered complete.

\subsection{Optimal Control for the Two-Track Model}

The optimal control solution for the two-track vehicle model of Section 2 is now considered. Because of the relative complexity of the model no analytical solution is attempted. Instead the problem is to be solved numerically for the brake forces, namely to find the optimal sequence of admissible brake inputs $F_{X i j}(t)$ subject to

$$
-\mu_{0} \mu_{i} F_{Z i j}(t) \leq F_{X i j}(t) \leq 0, \quad \forall t \in\left[0, T^{*}\right]
$$

where $\mu_{0}$ is the nominal road surface friction coefficient and $\mu_{i}$ is a friction coefficient specific to the particular axle.

The cost function Equation $(6)$ is to be minimized, while satisfying the equations of motion Equation (1), the initial conditions in Equation (7) and the constraint in Equation (8) at the final time $t=T$. The optimal control solver engine PROPT for Matlab [14] is used for this purpose. PROPT uses a pseudospectral Collocation method for solving optimal control problems. This method is a direct transcription method for discretizing a continuous optimal 
control problem into a nonlinear program [15. This means that the solution takes the form of a polynomial, and this polynomial satisfies the equations of motion Equation (1), constraints on the input variables Equation (9) and state variables Equation $(10)$ at the collocation points.

The following case is considered: $v_{0}=20 \mathrm{~m} / \mathrm{s}, \kappa_{\text {ref }}^{-1}=60 \mathrm{~m}$ and $\mu=0.4$, resulting in a limiting speed $v_{\lim }=15.3 \mathrm{~m} / \mathrm{s}$. Since the difference between the initial speed $v_{0}$ and the limiting speed $v_{\lim }$ is relatively large, precise tracking of the reference path is of course impossible. Results are shown (up to the point of maximum path deviation) in Figures 3 4 with comparisons to the PPR particle motion.

Figure 3 shows the trajectories in the inertial reference frame, where it can be seen that the optimal control for the two-track vehicle closely follows the PPR parabolic path. The maximum off-tracking is $8.6 \mathrm{~m}$ for the particle solution and $8.9 \mathrm{~m}$ for the two-track vehicle. Since the models and optimization procedures are entirely independent, this result confirms the broad validity of each solution. More significantly, it suggests that the optimal response to terminal understeer is dominated by controlling the acceleration vector at the mass center rather than purely through yaw moment control.

The match to PPR is confirmed from several other responses - Figure 4. In (a) the speed profiles are nearly identical, while in (c) and (d) it is seen the optimal control for the vehicle (OCV) finds the same inertially fixed acceleration vector as the particle (OCP). In (b) we note the large excursions in body sideslip angle $\beta$ arising from vehicle optimization, a point we return to in Section 4 , of course $\beta$ is not defined for the particle motion.

Control of the mass center acceleration vector, or equivalently the resultant 


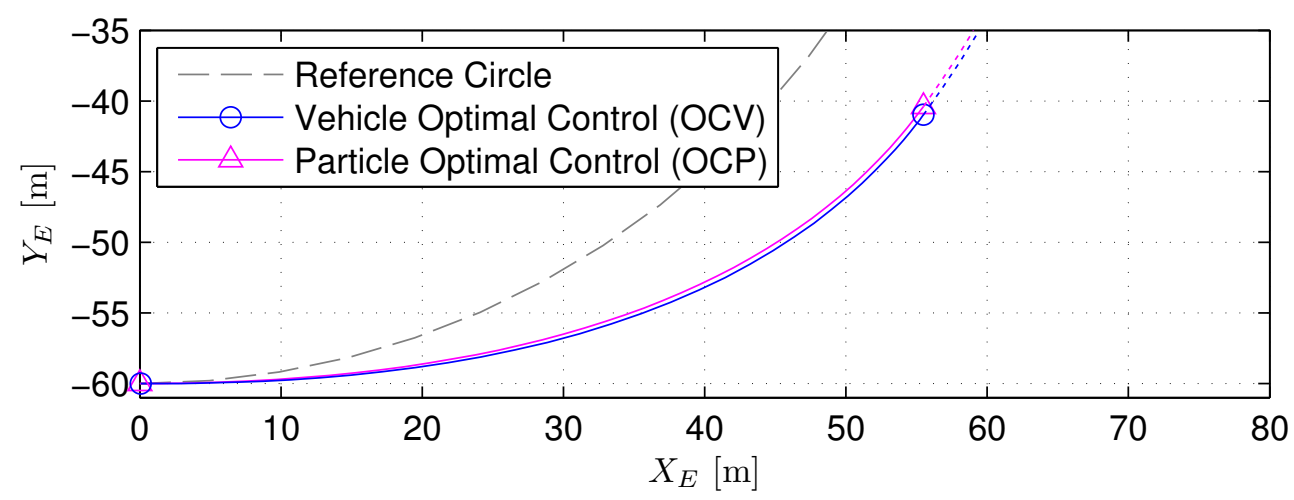

Figure 3: Optimal trajectories in the global $X_{E}, Y_{E}$ coordinate system for the two-track vehicle (OCV) and the particle (OCP). The maximum off-tracking is indicated with dotted arcs concentric with the reference path (dashed).

in-plane force vector, has been considered by other authors. The strategy in [9] targets a resultant control force at a body-fixed angle of $135^{\circ}$ in the vehiclefixed reference frame. In some scenarios this strategy may generate a greater lateral displacement from the direction of entry, but the maximum off-tracking is always larger than for the PPR solution.

Another case is the "G-vectoring" control described in [8], in which braking forces are applied proportional to the rate of change of the path lateral acceleration (lateral jerk). Here too, the mass center acceleration vector is directly adjusted according to the driver's steering input. The motivation of this approach is more in terms of driver feedback and reducing understeer by longitudinal load transfer, and no formal criteria are defined. One common feature between PPR and the two referenced approaches is that steering actions lead to controlled changes in speed. For PPR the magnitude of this effect is explicitly dependent on vehicle initial speed ( $v_{0}$ relative to $\left.v_{\text {lim }}\right)$, a property the other strategies do not share. 
(a)

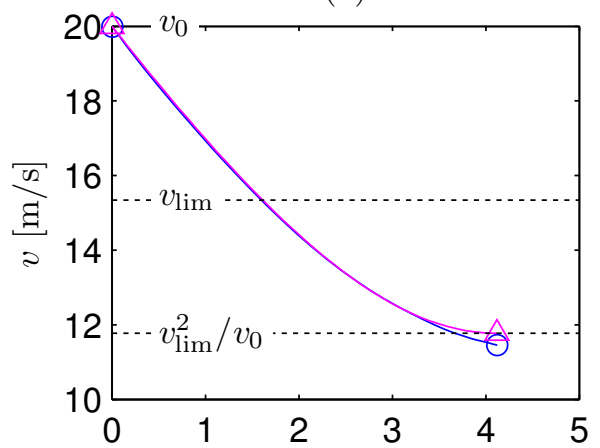

(c)

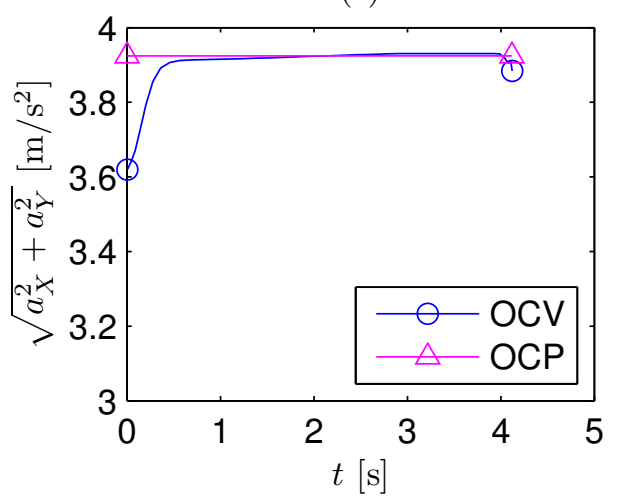

(b)

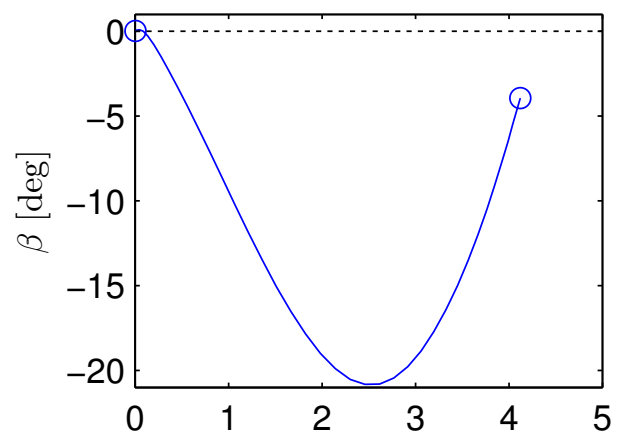

(d)

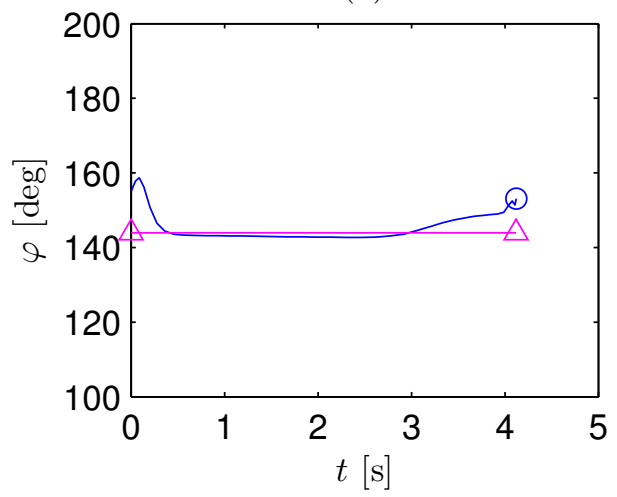

Figure 4: Optimal control results for the two-track vehicle (OCV) and the particle (OCP). Subplots show time histories of (a) speed, (b) sideslip angle (OCV only), (c) acceleration magnitude (d) the angle of the acceleration vector in the global reference frame. Note: the large sideslip angle in (b) may be reduced to acceptable levels without significantly affecting the path - see Table 3 


\section{Controller Synthesis and Evaluation}

The optimal control results are now used to formulate a candidate closed-loop controller, which we simply refer to as PPR, even though it is just one possible implementation of the general strategy. This is compared to the optimal offtracking performance of the vehicle model ( $\mathrm{OCV})$ presented above, as well as to the performance of a closed-loop controller based on yaw moment control (YC).

\subsection{Closed-loop Implementation of PPR}

It is proposed to use proportional feedback of the difference between the target speed obtained from the particle solution and the actual speed of the vehicle. By suitable tuning of the proportional gain, the speed profile can be fitted to that of the PPR reference; then, if the magnitude of the mass center acceleration is maintained at its limit, the overall acceleration vector is expected to follow the PPR reference. The target speed for the particle solution requires knowledge about the target curvature $\kappa_{\text {ref }}$ and the limit speed $v_{\text {lim }}$, which in turn requires an estimate of the surface friction. Since an intervention is only necessary when $v_{0}>v_{\text {lim }}$ this situation also implies that the friction limit is reached soon after

the steering input has been applied. In this case $\mu g \approx \sqrt{a_{Y}^{2}+a_{X}^{2}}$, which means that the friction coefficient can be estimated and thereby $v_{\lim }$ is determined from Equation (4).

The target speed is $v_{T} \equiv v\left(T^{*}\right)=v_{\lim }^{2} / v_{0}$ according to Equation (17). The proportional controller is to distribute the braking forces to the wheels, and hence there are four proportional gains, denoted $\gamma_{i j}$ :

$$
F_{X i j}(t)=-\gamma_{i j} m \max \left(v(t)-v_{T}, 0\right)
$$


Simple parameter optimization was used to select the gains in Equation 19 for best fit to the PPR speed profile; the following values were obtained: $\gamma_{11}=$ $0.115, \gamma_{12}=0.151, \gamma_{21}=0.081$ and $\gamma_{22}=0.114$. This implies larger braking forces at the front wheels, as would be expected. There is also a bias to increased braking on the outer wheels, where vertical load is higher, so the direct yaw moment from braking acts in the opposite sense to the turn direction; this is contrary to the standard yaw control strategy discussed in Section 1 suggesting that significant differences will be found when comparing PPR with YC. This turn-out yaw moment is beneficial for the yaw stability, although additional stabilizing yaw control would be necessary to account for disturbances.

\subsection{Understeer Mitigation by Yaw Control}

We consider a version of the "standard" yaw control (YC) strategy. No attempt is made in this paper to compare to all aspects of the understeer control of commercial stability control systems, engine intervention for instance, but only to compare the yaw control component, something that is most commonly referenced in the literature. The reason is to make the comparison clearer; for a comparison with an actual ESC systems we refer to experimental work reported in [11. The standard understeer mitigation proposed in the literature is to apply a turn-in yaw moment by braking the inner rear wheel. However, care must be taken not to over-brake the single wheel since this can lead to excessive sideslip [5]. Initial simulations determined that braking both inner wheels was more effective than braking only the inner rear wheel (which was also proposed in reference [7]), so this modification is implemented to improve the comparative

performance of YC. Thus for a left turn $(\dot{\psi}>0)$ the longitudinal force vector 
is:

$$
\left[\begin{array}{llll}
F_{X 11} & F_{X 12} & F_{X 21} & F_{X 22}
\end{array}\right]^{\top}=-K_{P 0}\left(\dot{\psi}_{\text {ref }}-\dot{\psi}\right)\left[\begin{array}{llll}
\eta_{0} & 0 & \left(1-\eta_{0}\right) & 0
\end{array}\right]^{\top}
$$

where, based on a neutral steered reference vehicle,

$$
\dot{\psi}_{\text {ref }}=v_{X} \kappa_{\text {ref }}
$$

Here $K_{P 0}>0$ and $0 \leq \eta_{0} \leq 1$ are tuning parameters, optimized in the same way as $\gamma_{i j}$ were optimized for PPR. The resulting parameter values are $K_{P 0}=18$ and $\eta_{0}=0.7$, giving a controller with similar performance to that presented in [7].

\subsection{Controller Evaluation}

Results for the two control strategies outlined above, together with the openloop OCV intervention, are shown in Figures 5-7. Again we consider the case with $v_{0}=20 \mathrm{~m} / \mathrm{s}, \kappa_{\text {ref }}^{-1}=60 \mathrm{~m}$ and $\mu=0.4$. Closed-loop PPR control is seen to give a close match to $\mathrm{OCV}$ in both path and speed profile - see Figure 5 and Figure 6 (a) respectively. PPR is shown throughout with diamond markers, while OCV is shown with circles. Subplots (c), (d) in Figure 6 show that the closed-loop controller does indeed follow the PPR reference for mass center acceleration, approximating an inertially fixed mass center acceleration operating at the friction limit and with the desired global direction. Thus the simple algorithm based on speed tracking seems sufficient for the proposed comparisons.

While PPR does not include any specific yaw control strategy it is seen in Figure 6 (e), (f) that very similar yaw accelerations exist between PPR and OCV. Figure 6 (e) includes only the direct contributions from braking forces 


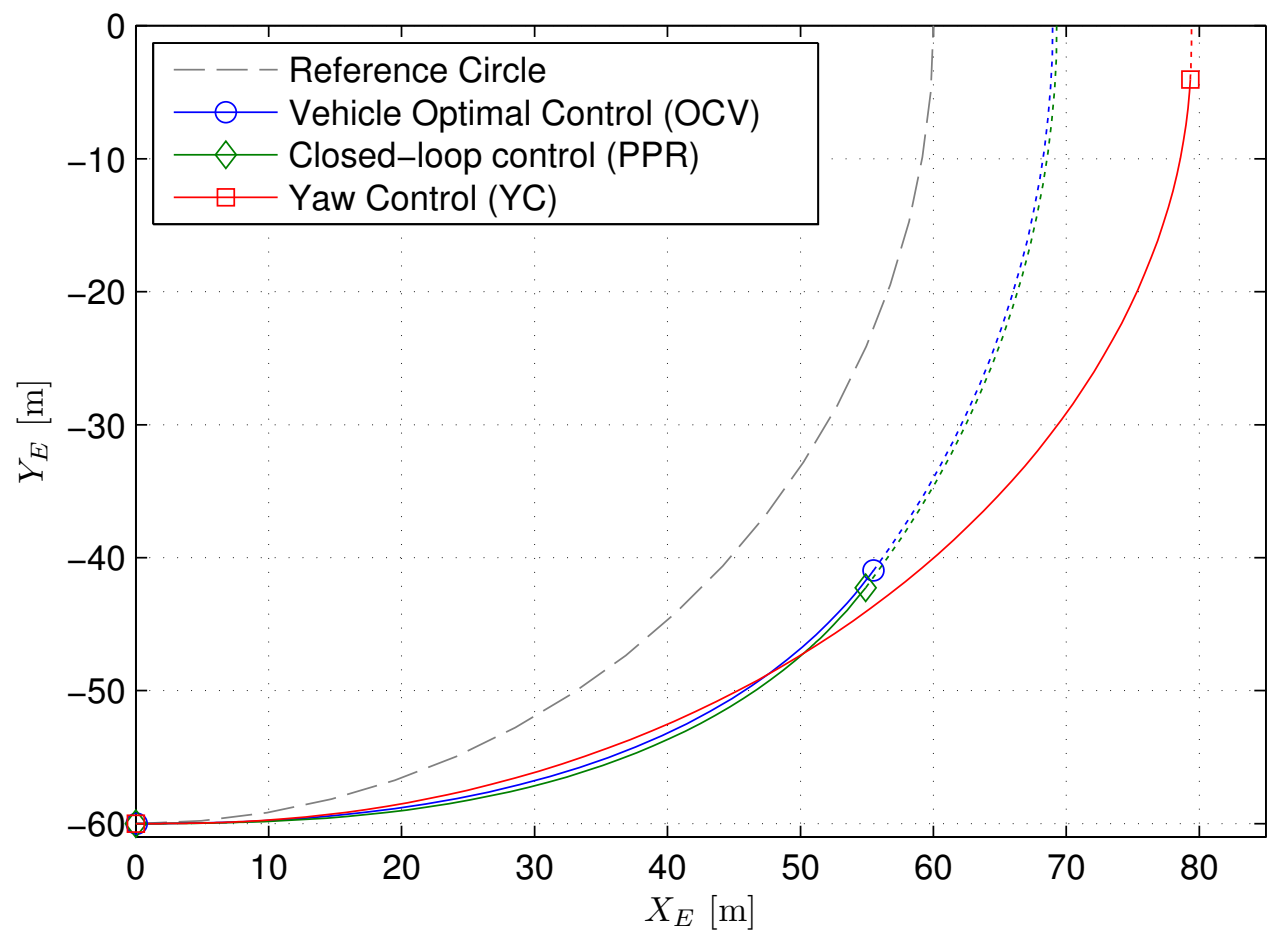

Figure 5: Trajectories in global $X_{E}, Y_{E}$ coordinates: optimized two-track vehicle (OCV), closed-loop PPR strategy (PPR) and yaw control (YC)

$F_{X i j}$ and the two cases give consistent negative yaw accelerations, i.e. as mentioned above there is a consistent turn-out yaw moment arising from braking forces.

Individual wheel braking forces are shown in Figure 7 where again there is a high degree of consistency between PPR and OCV; this includes the common bias towards front wheel braking and a slight bias towards increased force at the outer wheels. In fact it can be seen that PPR does not require any complex actuator inputs to achieve the desired results. The plot with greatest discrepancy between PPR and OCV is in body sideslip, Figure 6(b). In this case the peak angle $\beta_{\max }$ is reduced from approximately $20^{\circ}$ to $15^{\circ}$ in the closed-loop. 
(a)

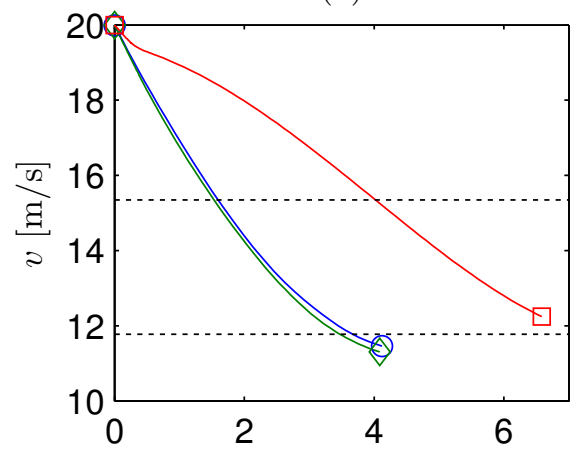

(c)

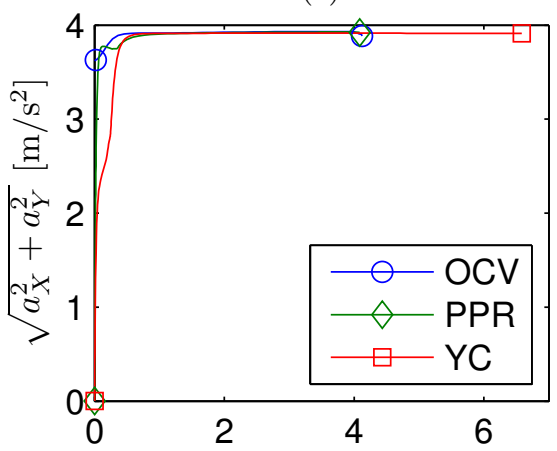

(e)

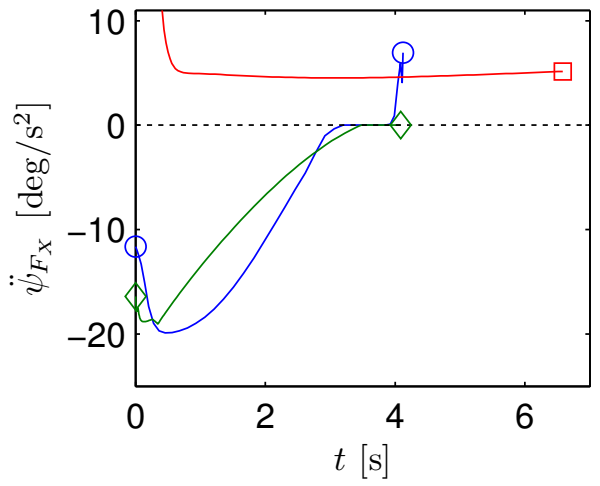

(b)

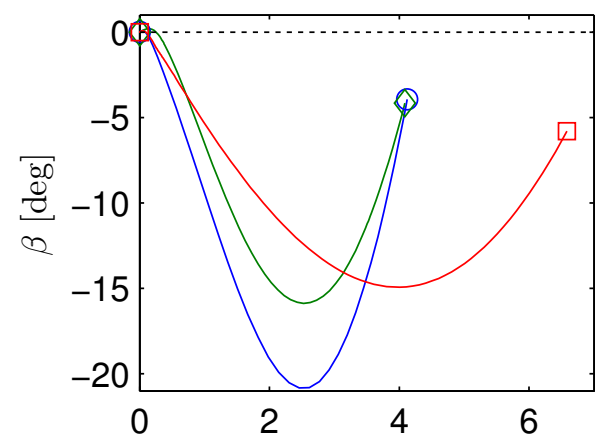

(d)

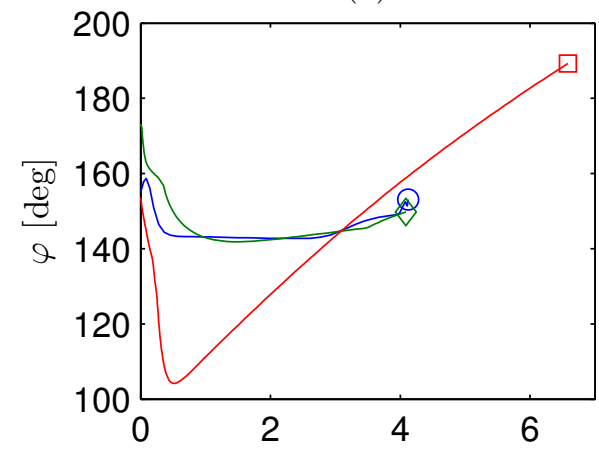

(f)

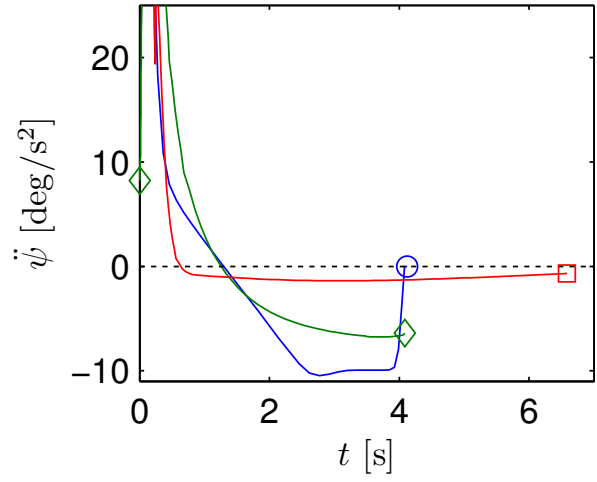

Figure 6: Time history responses of selected vehicle variables: (a) vehicle speed; (b) body sideslip angle; (c) resultant acceleration magnitude; (d) direction of the acceleration vector in global coordinates; (e) yaw acceleration due to brake forces; (f) vehicle yaw acceleration 
(a)

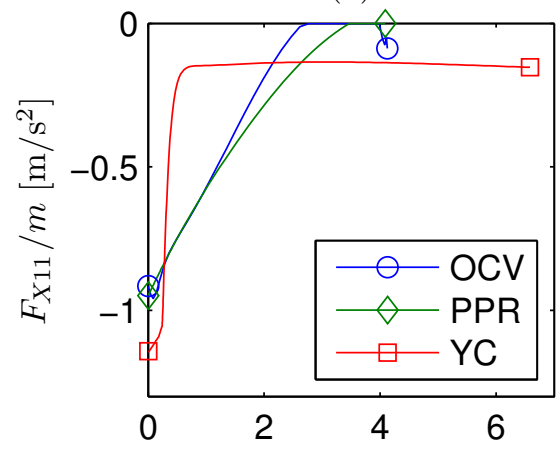

(c)

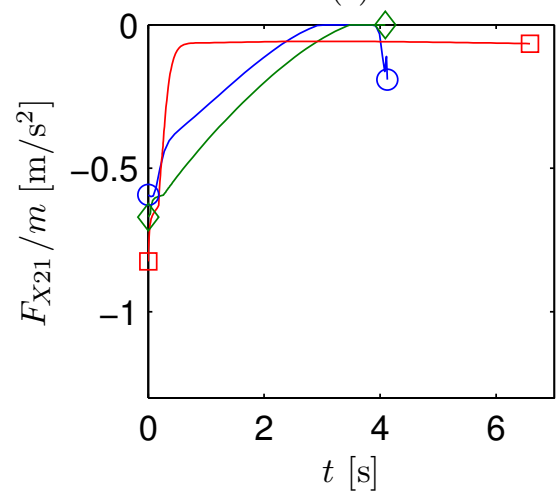

(b)

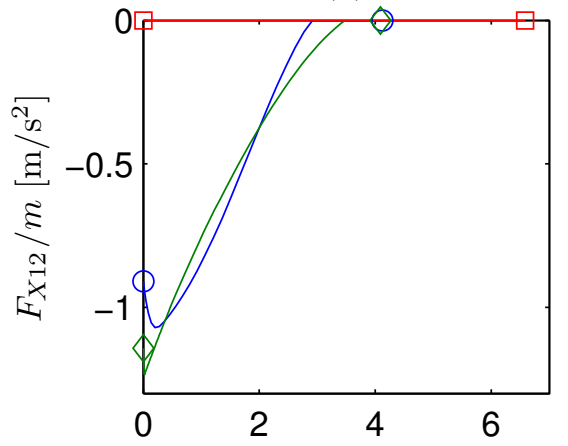

(d)

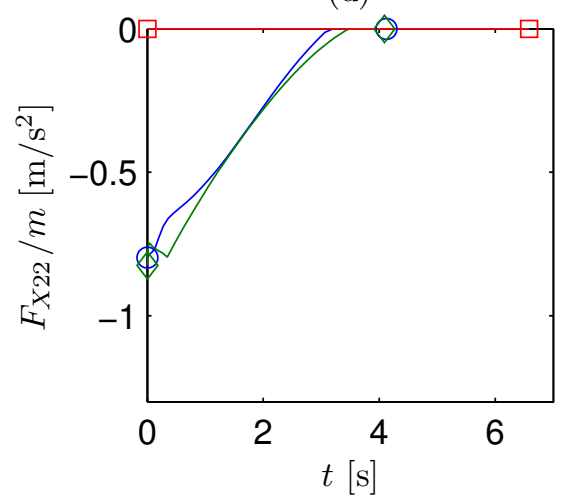

Figure 7: Control inputs: each sub-plot shows the corresponding longitudinal tyre force $F_{X i j}$ normalized by vehicle mass 
The fact that other responses are hardly affected also suggests that $\beta_{\max }$ might be further constrained without significantly degrading the performance relative to the PPR reference - we return to this point.

The comparison with YC (square marker) is shown in the same figures. Maximum off-tracking is significantly greater than for PPR, and while speed reductions are quite similar by the end of the intervention, the mean deceleration is a lot less for YC. According to Figure $6 \beta_{\max }$ is also similar to that of PPR. In Figure 6 (d) the angle $\varphi$ for $\mathrm{YC}$ is not constant; it corresponds to an acceleration vector that is approximately fixed with respect to the vehicle path; while PPR (and OCV) shows a strong deceleration bias initially, transitioning to path-lateral acceleration as the point of maximum deviation is approached, YC persists in emphasizing path-lateral acceleration with reduced longitudinal deceleration. As should be clear now, this extends the period of time for the intervention and leads to larger overall off-tracking.

In Figure 6 (e) we see the yaw accelerations due to longitudinal tire forces, hence representing the direct yaw moment from brake control; the most obvious difference is in the difference in sign of PPR, which creates a turn-in yaw moment, as of course was prescribed. Also, Figure 7, we see that for $\mathrm{YC}$, the brake intervention has two phases, with an initial sharp brake pulse followed by much reduced braking effort. This highlights the fact that $\mathrm{YC}$ operates mostly via the passive lateral tire forces, the strong transient creating a turn-in yaw moment to rapidly increase slip angles at the rear tires.

This is in contrast with PPR/OCV, for which deceleration is initially the major goal, smoothly transitioning to path curvature as the priority. Figure 6 (f) shows the total yaw moment, including the effects of lateral tire forces; in 
all cases there is an initial turn-in moment followed by a correcting turn-out moment. For YR the effect is something like a switch, where a turn-in pulse is later compensated by a small but constant turn-out in quasi-steady-state. Figure 7 shows the same comparison with a discrete switch in YR brake forces, compared to the progressive transition for PPR/OCV. It is worth noting that the increased overall braking effort in PPR has the added advantage of increasing the vertical load on the front tires, thus increasing the available lateral tire forces at the front axle.

Here the $\mathrm{YC}$ controller does not include oversteer mitigation, as is the case with the other controllers; this is to avoid confounding off-tracking performance with a possible tradeoffs due to yaw instability intervention. It could be argued that $\mathrm{YC}$ performance can be improved if oversteer correction is indeed included, leading to subsequent braking of the outer wheels and further deceleration. Since the focus of this study is on the underlying control concepts it does not seem appropriate to probe the extent to which the addition of further control rules can be used to improve the off-tracking performance of YC.

Off-tracking comparisons for multiple cases are summarized in Table 2, where the second row is the case considered in detail above $\left(v_{0}=20 \mathrm{~m} / \mathrm{s}, \kappa_{\mathrm{ref}}^{-1}=60 \mathrm{~m}\right.$, $\mu=0.4$ ). The theoretical particle results (OCP) are included for comparison, and in all cases the closed-loop PPR performs similar to OCV and the relative off-tracking performance of $\mathrm{YC}$ is significantly worse. It is also noticeable that the vehicle results diverge significantly from the OCP particle only when $v_{0}$ is close to $v_{\text {lim }}$ - in this case the time horizon for control is smaller, and hence the transient delay due to yaw inertia is more influential.

In the above no state constraints Equation 100 were imposed, but, because 


\begin{tabular}{cccc|cccc} 
& & & & \multicolumn{5}{|c}{$\varepsilon_{\max }[\mathrm{m}]$} \\
$v_{0}[\mathrm{~m} / \mathrm{s}]$ & $v_{\text {lim }}[\mathrm{m} / \mathrm{s}]$ & $\kappa_{\text {ref }}^{-1}[\mathrm{~m}]$ & $\mu$ & OCP & OCV & PPR & YC \\
\hline 16 & 15.3 & 60 & 0.4 & 0.2 & 0.61 & 0.8 & 2.0 \\
20 & 15.3 & 60 & 0.4 & 8.6 & 8.97 & 9.3 & 19.6 \\
25 & 15.3 & 60 & 0.4 & 30.9 & 31.3 & 32.8 & 50.3 \\
\hline 25 & 21.7 & 120 & 0.4 & 4.8 & 5.84 & 6.1 & 9.8 \\
30 & 21.7 & 120 & 0.4 & 26.1 & 26.9 & 27.7 & 40.8 \\
\hline 25 & 21.7 & 60 & 0.8 & 2.4 & 2.9 & 3.7 & 8.1 \\
35 & 21.7 & 60 & 0.8 & 29.6 & 29.6 & 33.1 & 49.4
\end{tabular}

Table 2: Off-tracking results for variations in the initial speed, road friction and target radius.

of the large body sideslip observed in the response, it was decided to rerun the numerical optimization with the additional constraint $|\beta(t)| \leq 5^{\circ}$. Results are shown in Table 3 . For each scenario the second entry gives the result with constraint applied and in every case $\max (|\beta(t)|)=5^{\circ}$, i.e. the constraint becomes active during the intervention. This leads to large reductions in $\max (|\beta(t)|)$ in many cases, but with very little effect on $\varepsilon_{\max }$. This result appears significant: yaw control may be applied in a way that does not seriously degrade the underlying PPR strategy, even to the point where we can consider the path control (implementation of PPR) to be largely decoupled from the required residual yaw control (adjusting yaw moments to limit sideslip).

The reason for the relative insensitivity to maximum side-slip angle in our simulations is understood to be due to fact that there are mulitple ways to realize the necessary global force vector with different combinations of tire lateral 


\begin{tabular}{|c|c|c|c|c|}
\hline$v_{0}[\mathrm{~m} / \mathrm{s}]$ & $v_{\lim }[\mathrm{m} / \mathrm{s}]$ & $\kappa_{\text {ref }}^{-1}[\mathrm{~m}]$ & $\varepsilon_{\max }[\mathrm{m}]$ & $\max (|\beta(t)|)\left[^{\circ}\right]$ \\
\hline \multirow[t]{2}{*}{16} & 15.3 & 60 & 0.61 & 5.6 \\
\hline & & & $0.61^{*}$ & 5.0 \\
\hline \multirow[t]{2}{*}{20} & 15.3 & 60 & 8.97 & 20.8 \\
\hline & & & $9.05^{*}$ & 5.0 \\
\hline \multirow[t]{2}{*}{25} & 15.3 & 60 & 31.3 & 37.8 \\
\hline & & & $31.4^{*}$ & 5.0 \\
\hline \multirow[t]{2}{*}{25} & 21.7 & 120 & 5.84 & 15.9 \\
\hline & & & $5.92^{*}$ & 5.0 \\
\hline \multirow[t]{2}{*}{30} & 21.7 & 120 & 26.9 & 29.9 \\
\hline & & & $27.1^{*}$ & 5.0 \\
\hline
\end{tabular}

Table 3: Off-tracking and maximum sideslip for the optimal vehicle response (OCV) - without and with* sideslip constraint imposed.

and longitudinal forces. Although only braking actions are available to the controller, provided all tires have a large enough slip angle to saturate the tire laterally, the brake forces can influence the direction of the force vector on each wheel within wide limits - from perpendicular to the wheel rolling direction in the free rolling case, to a direction opposite to velocity vector under wheel lockup.

\section{Conclusions}

This paper has considered the problem of a vehicle overshooting a reference trajectory due to friction limits, a situation referred to as terminal understeer. In order to minimize the effects of a deviation from the desired trajectory, the 
recovery task has been formulated as an optimal control problem: to minimize the maximum off-tracking distance from the reference trajectory.

For any given recovery from terminal understeer strategy, maximum offtracking occurs when the velocity vector is tangent to the reference trajectory. In this work, the particular case of a circular reference trajectory has been considered, and a rigorous optimal control strategy has been found for a particle with bounded acceleration magnitude.

It was found that minimization of maximum off-tracking is achieved by directing the force in a globally fixed direction, perpendicular to the path tangent at the anticipated point of maximum off-tracking. The optimal recovery from terminal understeer to the reference trajectory is identified as a parabolic motion, familiar from the motion of an ideal projectile under gravity.

It was found that the new strategy solves the vehicle understeer problem with significantly reduced maximum off-tracking and without requiring an unbalancing moment that may compromise yaw stability. Although yaw stability remains an important aspect of the understeer mitigation, the results imply that control of the mass center acceleration vector is most effective for limiting the path deviations. Also, it has been found that a turn-out yaw moment from the direct braking action can be more successful than a turn-in yaw moment as this is associated with higher initial deceleration.

Robust implementation of the proposed control will require further development, especially as a closed-loop control strategy that directs the global mass center acceleration vector according to the PPR reference. As mentioned, preliminary experiments [11] show promising possibility to implement PPR within a robust integrated chassis controller. Of course it is possible that path off- 
tracking may occur simultaneously with yaw instability, in which case coordination or arbitration between PPR and yaw stabilization will be required. Numerical solutions using a two-track model, however, indicate that similar solutions in terms of maximum off-tracking can be achieved with largerly different maximum side-slip angles. This indicates that, although important for yaw stability, controlling the side-slip angle is less critical to the path control problem considered. This in turn may lead to the conclusion that PPR and existing yaw stability control could be combined without seriously degrading either function.

In this paper the path curvature has been inferred from the driver input, which is an assumption with limitations, but in the future it is possible that vehicle sensors, digital maps and/or wireless communication can provide the vehicle motion controller with additional information to update the reference trajectory, so the driver interpretation function may become less critical for path planning in certain cases. Two particularly relevant examples are: path planning for autonomous vehicles and accident avoidance maneuvers. Although our experimental work reported in [1] did study the effects of driver interaction, especially including the effect of the driver applying additional corrective steer angles as off-tracking is perceived, more work on this topic is likely warranted.

Overall, the main contribution of this paper is that whenever vehicle speed and surface friction are incompatible with the target path, and within the approximations used, the control strategy presented minimizes the maximum offtracking and provides for improved vehicle safety. In future work the authors plan to implement and evaluate the new control strategy considering yaw stability, general reference trajectories, more sophisticated vehicle dynamics models and further tests with an experimental vehicle. 


\section{Acknowledgments}

The authors wish to thank the Swedish national research program Intelligent Vehicle Safety Systems (IVSS) and the SAFER vehicle and traffic safety center at Chalmers for financial support. Further the authors would like to acknowledge the valuable discussions and input to our work from Anders Boström and Bo Egardt from Chalmers University of Technology as well as Fredrik Wrang and Gunnar Olsson from Saab Automobile.

\section{References}

(1) Liu C, Subramanian R. Factors Related to Fatal Single-Vehicle Run-OffRoad Crashes. NHTSA DOT HS 811 232; 2009.

(2) Liu C, Ye TJ. Run-Off-Road Crashes: An On-Scene Perspective. NHTSA DOT HS 811 500; 2011.

(3) Shibahata Y, Shimada K, Tomari T. Improvement of Vehicle Maneuverability by Direct Yaw Moment Control. Vehicle System Dynamics. 1993;22:465-481.

(4) van Zanten A, Erhardt R, Pfaff G, Kost F. Control aspects of the BoschVDC. In: Proc. AVEC, Aachen; 1996. .

(5) Ghoneim YA, Lin WC, Sidlosky DM, Chen HH, Chin YK, Tedrake MJ. Integrated chassis control system to enhance vehicle stability. International Journal of Vehicle Design. 2000;23:124-144.

(6) NHSTA. Electronic Stability Control Systems. FMVSS No. 126 - Final Regulatory Impact Analysis; 2007. 
(7) Antonov S. Model-based Vehicle Dynamics Control. Shaker Verlag, Aachen; 2008.

(8) Yamakado M, Takahashi J, Saito S, Yokoyama A, Abe M. Improvement in vehicle agility and stability by G-Vectoring control. Vehicle System Dynamics. 2010;48(sup1):231-254.

(9) Blank M, Margolis DL. Minimizing the Path Radius of Curvature for Collision Avoidance. Vehicle System Dynamics. 2000;33(3):183-201.

(10) Nozad A, Lidberg M, Gordon T, Klomp M. Optimal Path Recovery from Terminal Understeer. In: IAVSD Symposium, Manchester, UK; 2011. .

(11) Gordon TJ, Klomp M, Lidberg M. Strategies for Minimizing Maximum Off-tracking resulting from Over-speed in Curves. In: Proceedings of 11th International Symposium on Advanced Vehicle Control (AVEC), Seoul Korea; 2012. .

(12) Klomp M. Longitudinal force distribution using quadratically constrained linear programming. Vehicle System Dynamics. 2011;49(12):1823-1836.

(13) Pacejka HB. Tyre and Vehicle Dynamics. 2nd ed. Butterworth-Heinemann, Oxford, UK; 2006.

(14) Rutquist PE, Edvall MM. PROPT - Matlab Optimal Control Software. 1260 SE Bishop Blvd Ste E, Pullman, WA 99163, USA: Tomlab Optimization Inc.; 2010.

(15) Fahroo F, Ross IM. Trajectory Optimization by a Chebyshev Pseudospectral Method. Journal of Guidance, Control, and Dynamics. 2002;25:160166. 
(16) Bryson AE, Ho YC. Applied Optimal Control. Hemisphere Pub. Corp., USA; 1975.

\section{A Tire Model}

The lateral forces, $F_{Y i j}$, are in this paper modeled as function of the tyre slip angles on each wheel, $\alpha_{i j}$, the longitudinal forces, $F_{X i j}$, and vertical forces, $F_{Z i j}$, by using a simple saturation tyre model, where

$$
F_{Y i j}=D_{Y i j} \tanh \left(C_{Y} B_{Y} \alpha_{i j}\right)
$$

where

$$
D_{Y i j}=\sqrt{\left(\mu_{0} \mu_{i} F_{Z i j}\right)^{2}-F_{X i j}^{2}}, \quad C_{Y}=3 / 2, \quad B_{Y}=10 / \mu_{0}
$$

where $i=1,2$ for the front and rear axle respectively and where $\mu_{0}$ is the nominal road friction. The slip angles, $\alpha_{i j}$, are related to the longitudinal, lateral and yaw velocity at the center of gravity as

$$
\alpha_{i j}=\delta_{i}-\arctan \frac{v_{Y}-(-1)^{i} l_{i} \dot{\psi}}{\left|v_{X}+(-1)^{j} s \dot{\psi}\right|}
$$

which is the angle between direction of the tyre velocity vector and the free rolling direction using the definition in [13].

\section{B Vehicle Data}

The vehicle data shown in Table 4 that are used in the conducted simulations represent a medium-sized passenger vehicle. 


\begin{tabular}{|l|c|c|}
\hline Description & Symb. & Value \\
\hline \hline Vehicle mass [kg] & $m$ & 1675 \\
\hline Yaw radius of gyration [m] & $k$ & 1.32 \\
\hline Wheel base [m] & $l$ & 2.675 \\
\hline Distance from axle to mass center (front/rear) [m] & $l_{1} / l_{2}$ & $0.4 l / 0.6 l$ \\
\hline Track width (f/r) [m] & $w$ & 1.5 \\
\hline Mass center height [m] & $h$ & 0.5 \\
\hline Longitudinal load transfer coefficient [-] & $\zeta_{X}$ & $h /(2 l)$ \\
\hline Lateral load transfer coefficient (f/r) [-] & $\zeta_{Y 1} / \zeta_{Y 2}$ & $0.17 / 0.16$ \\
\hline Road friction coefficient [-] & $\mu_{0}$ & 0.4 \\
\hline Axle friction coefficients (f/r) [-] & $\mu_{1} / \mu_{2}$ & $0.97 / 1.05$ \\
\hline
\end{tabular}

Table 4: Vehicle Data

\section{Optimal Control Proof for the Particle Repre-}

\section{sentation}

In order to aid the proof we re-write Equation (11) to state-space form:

$$
\dot{\mathbf{x}}=\mathbf{A x}+\mathrm{q}(\gamma), \quad \forall \gamma \in \mathcal{U}
$$

where the state vector, $\mathbf{x}$, and input vector, $\gamma$, are

$$
\begin{aligned}
& \mathbf{x}=\left[\begin{array}{llll}
X_{E} & Y_{E} & \dot{X}_{E} & \dot{Y}_{E}
\end{array}\right]^{\top} \\
& \gamma=\left[\begin{array}{ll}
F & \varphi
\end{array}\right]^{\top}
\end{aligned}
$$


The state matrix, A, and the input function, $\mathrm{q}(\gamma)$, are

$$
\begin{aligned}
\mathbf{A} & =\left[\begin{array}{ll}
\mathbf{0}_{2 \times 2} & \mathbf{I}_{2 \times 2} \\
\mathbf{0}_{2 \times 2} & \mathbf{0}_{2 \times 2}
\end{array}\right] \\
\mathrm{q}(\gamma)= & \frac{F}{m}\left[\begin{array}{c}
\mathbf{0}_{2,1} \\
\cos \varphi \\
\sin \varphi
\end{array}\right]
\end{aligned}
$$

where $\mathbf{0}_{2 \times 2}$ is a $2 \times 2$ zero matrix.

\section{C.1 Problem}

The optimal recovery from terminal understeer to minimize the first maximum value of $\varepsilon$ is formulated as a free-time optimal control problem [16]. This problem is to find the admissible control $\gamma^{*}$ and a feasible trajectory $\mathrm{x}^{*}$ that minimizes the maximum squared radial distance from the center of the reference circle. As in Section 3 this in turn is formulated as minimizing the terminal cost

$$
J=X_{E}^{2}(T)+Y_{E}^{2}(T)
$$

Additionally the solution is subject to the initial and terminal conditions

$$
\begin{array}{r}
\mathbf{x}_{0}=\left[\begin{array}{llll}
0 & -\kappa_{\text {ref }} & v_{0} & 0
\end{array}\right]^{\top} \\
X_{E}(T) \dot{X}_{E}(T)+Y_{E}(T) \dot{Y}_{E}(T)=0
\end{array}
$$

Admissible control inputs (12) are $\gamma \in \mathcal{U}$ and admissible trajectories satisfies the dynamic model (25) and initial/terminal conditions (28). The control $\gamma^{*}$ is the optimal control and the corresponding trajectory $\mathrm{x}^{*}$ is the optimal trajectory. The terminal condition ensures that the terminal cost $J$ minimizes the maximum off-tracking, not the off-tracking at an arbitrary final time $T$. 


\section{C.2 Method}

The optimal control problem for the particle representation is solved by extending the dynamical model (25) to an augmented Hamiltonian system

$$
\left\{\begin{array}{l}
\dot{\mathbf{x}}=\partial H / \partial \lambda \\
\dot{\lambda}=-\partial H / \partial \mathbf{x}
\end{array}\right.
$$

where $\lambda$ is the co-state vector and where the Hamiltonian function is

$$
H=\lambda^{\top}(\mathbf{A x}+\mathrm{q}(\gamma))
$$

According to Pontryagin's minimum principle the Hamiltonian must be minimized over the set of all permissable controls, $\mathcal{U}$, satisfying all constraints. If $\gamma^{*} \in \mathcal{U}$ is the optimal control for the problem, then the principle states that

$$
H\left(\mathbf{x}^{*}, \gamma^{*}, \lambda^{*}\right) \leq H(\mathbf{x}, \gamma, \lambda)
$$

Additionally the transversality conditions require that

$$
\lambda^{*}(T)=\left.\frac{\partial J}{\partial \mathbf{x}}\right|_{t=T}
$$

When a solution that minimizes Equation $(6)$ is found for an arbitrary $T$, the final step is to separately determine $T^{*}$, such that the terminal condition in Equation $(28)$ is satisfied.

\section{C.3 Solution}

From the Hamiltonian $(30)$ we obtain that

$$
H=\lambda_{1} x_{1}+\lambda_{2} x_{2}+F / m\left(\lambda_{3} \cos \varphi+\lambda_{4} \sin \varphi\right)
$$

Since $F$ appears linearly in Equation (33), and taking Equation 12 into account, we have from [16] that $H$ is minimized if

$$
F^{*} \equiv \mu m g,
$$


noting that the direction of the force is only determined by the angle $\varphi$. The optimal angle, $\varphi^{*}$, is found by knowing that at the extremal of $H$

$$
\frac{\partial H}{\partial \varphi}=F / m\left(\lambda_{3} \sin \varphi-\lambda_{4} \cos \varphi\right)=0
$$

which gives that

$$
\tan \varphi^{*}=\frac{\lambda_{4}}{\lambda_{3}}
$$

In order to determine $\lambda_{3}$ and $\lambda_{4}$, we obtain from Equation $(29)$ that

$$
\dot{\lambda}=-\mathbf{A} \lambda=\left[\begin{array}{llll}
0 & 0 & -\lambda_{1} & -\lambda_{2}
\end{array}\right]^{\top}
$$

which, after integration, gives that

$$
\lambda(t)=\left[\begin{array}{llll}
C_{1} & C_{2} & -C_{1} t+C_{3} & -C_{2} t+C_{4}
\end{array}\right]^{\top}
$$

where $C_{1}, C_{2}, C_{3}$ and $C_{4}$ are integration constants. From Equation 32 we have that

$$
\lambda(T)=\left[X_{E}(T) \quad Y_{E}(T) \quad 0 \quad 00\right]^{\top}
$$

When Equation $(39)$ is combined with Equation $(38)$ the integration constants are determined to

$$
\left[\begin{array}{llll}
C_{1} & C_{2} & C_{3} & C_{4}
\end{array}\right]=\left[\begin{array}{llll}
X_{E}(T) & Y_{E}(T) & T X_{E}(T) & T Y_{E}(T)
\end{array}\right]
$$

which, when combined with Equation (38), gives that

$$
\lambda(t)=\left[\begin{array}{llll}
X_{E}(T) & Y_{E}(T) & (T-t) X_{E}(T) & (T-t) Y_{E}(T)
\end{array}\right]^{\top}
$$

Combining Equations (36) and (41) we obtain that

$$
\varphi^{*}=-\arctan \frac{Y_{E}(T)}{X_{E}(T)}+\pi
$$

The optimal control 34 and 42 is valid for any arbitrary final time $T$ that yields a feasible solution. Since we would like to minimize the maximum 
off-tracking, we will choose the final time, $T$, such that the terminal condition given in Equation (28) is satisfied. Therefore, by combining Equations (28) and 42 , we obtain that

$$
\varphi^{*}=\nu_{T}+\pi / 2
$$

where $\nu_{T}=\nu(T)$.

It follows from the terminal condition $(28)$ that the velocity perpendicular to a plane defined by the angle $\nu_{T}$ is zero at $t=T$. The optimal final time, $T^{*}$, is obtained by integration of the model $(25)$ in the direction perpendicular to this $\nu_{T}$-plane and initial values (7), such that

$$
T^{*}=\frac{v_{0}}{\mu g} \sin \nu_{T}
$$

The angle $\nu_{T}$ can now be determined by the distance traveled parallel to the $\nu_{T^{-}}$ plane and the radius of the reference circle. This parallel distance is determined by double integration of the model (25) with initial values (7) and Equation (44), from which we obtain that

$$
\cos \nu_{T}=v_{0} T \kappa_{\mathrm{ref}} \sin \nu_{T}=\frac{v_{\mathrm{lim}}^{2}}{v_{0}^{2}}
$$

Summarizing, we find by combining Equations (34), (42), (44) and (45), that the optimal control input $\gamma^{*}$ is

$$
\gamma^{*}=\left[\begin{array}{c}
\mu m g \\
\arccos \left(v_{\lim }^{2} / v_{0}^{2}\right)+\pi / 2
\end{array}\right] \quad \forall t \in\left[0, T^{*}\right]
$$

The optimal final time, $T^{*}$, expressed in the initial values is found by combining Equations (44) and 45), such that

$$
T^{*}=\frac{v_{0}}{\kappa_{\mathrm{ref}} v_{\lim }^{2}} \sqrt{1-\frac{v_{\mathrm{lim}}^{4}}{v_{0}^{4}}}
$$

